Discrete Comput Geom 38:513-525 (2007)

DOI: $10.1007 / \mathrm{s} 00454-007-1355-2$

\title{
Tverberg's Transversal Conjecture and Analogues of Nonembeddability Theorems for Transversals*
}

\author{
Roman N. Karasev \\ Department of Mathematics, \\ Moscow Institute of Physics and Technology, \\ Institutskiy per. 9, Dolgoprudny, Russia 141700 \\ r_n_karasev@mail.ru
}

\begin{abstract}
In this paper we prove a special case of the transversal conjecture of Tverberg and Vrećica. We consider the case when the numbers of parts $r_{i}$ in this conjecture are powers of the same prime. We also prove some results on common transversals that generalize the classical nonembeddability theorems.

We also prove an analogue of the colored Tverberg's theorem by Živaljević and Vrećica. Instead of multicolor simplices with a common point it gives multicolor simplices with a common $m$-transversal.
\end{abstract}

\section{Tverberg's Transversal Conjecture}

In this paper we prove a special case of the transversal conjecture of Tverberg and Vrećica.

Conjecture 1. Let $0 \leq m \leq d-1$ and let $S_{0}, S_{1}, \ldots, S_{m}$ be $m+1$ finite sets in $\mathbb{R}^{d}$. Let $\left|S_{i}\right|=\left(r_{i}-1\right)(d-m+1)+1$. Then every set $S_{i}$ can be partitioned into $r_{i}$ parts $S_{i 1}, S_{i 2}, \ldots, S_{i r_{i}}$ so that all the sets conv $S_{i j}$ can be met by a single $m$-flat.

This conjecture was formulated by Tverberg at the 1989 Symposium on Combinatorics and Geometry in Stockholm. In print it was first formulated by Tverberg and Vrećica in [8], where a special case of this conjecture was proved.

In [15] and [11] Živaljević and Vrećica established the case of this conjecture when all $r_{i}$ are equal to the same prime $p$, if $p$ is odd then $d$ and $m$ were also required to be odd.

* This research was supported by the Russian Foundation for Basic Research, Grant No. 06-01-00648, and by the President of the Russian Federation, Grant No. MK-5724.2006.1. 
Here we prove the theorem that generalizes the results of Živaljević and Vrećica to prime powers and show that the condition that $d$ is odd is not necessary. Our proof is quite short because we use the multiplicative rule for the Euler class. We prove that this conjecture is true when the numbers $r_{i}$ are powers of the same prime $r_{i}=p^{k_{i}}$, and for odd $p$, we also need $d-m$ to be even. Similarly to what was done by Živaljević and Vrećica, we prove a more general topological version of this conjecture.

Theorem 1. Let $0 \leq m \leq d-1$, let $r_{i}(i=0, \ldots, m)$ be powers of the same prime $r_{i}=p^{k_{i}}$. If $p$ is odd, let $d-m$ be even. Let, for each $i=0, \ldots, m, f_{i}$ map continuously an $\left(r_{i}-1\right)(d-m+1)$-dimensional simplex $\Delta_{i}=\Delta^{\left(r_{i}-1\right)(d-m+1)}$ to $\mathbb{R}^{d}$. Then every simplex $\Delta_{i}$ has $r_{i}$ points $x_{i 1}, x_{i 2}, \ldots, x_{i r_{i}} \in \Delta_{i}$ with pairwise disjoint supports so that all the points $f_{i}\left(x_{i j}\right)$ are contained in a single $m$-flat.

\section{Transversal Analogues of Nonembeddability Theorems}

In this paper we generalize some results of [15] that give transversal analogues of nonembeddability theorems. We have to recall some basic concepts in equivariant topology, for a detailed explanation see Chapters III and IV of [5].

Let us take the classifying $G$-space $E G$ and define the $G$-equivariant cohomology of a $G$-space $X$ as

$$
H_{G}^{*}(X, K)=H^{*}((X \times E G) / G, K) .
$$

Now consider the case $G=Z_{2}$. The cohomology ring of the one-point space $\Lambda=$ $H_{Z_{2}}^{*}$ (pt, $Z_{2}$ ) can be represented as $\Lambda=Z_{2}[u]$, where $\operatorname{dim} u=1$. If $Z_{2}$ acts on $\mathbb{R}^{n}$ by sending $x$ to $-x$, then the equivariant Euler class of this representation is

$$
e\left(\mathbb{R}^{n}\right)=u^{n} \in H_{Z_{2}}^{n}\left(\mathrm{pt}, Z_{2}\right) .
$$

For any space $Y$ with a $Z_{2}$ action the equivariant map $Y \rightarrow$ pt gives the canonical map $\Lambda \rightarrow H_{Z_{2}}^{*}\left(Y, Z_{2}\right)$. If this does not lead to confusion, we denote the images of $u^{k}$ under this map by $u^{k}$.

Definition. Let $Y$ be a $Z_{2}$-space. The maximal $n$ such that $u^{n} \neq 0 \in H_{Z_{2}}^{n}\left(Y, Z_{2}\right)$ (it may be $+\infty$ ) is called the homological index of $Y$. We denote it hind $Y$.

For any topological space we denote $X_{\Delta}^{2}=X \times X \backslash \Delta(X)$ as the deleted product of $X$, i.e. the square of $X$ without the diagonal.

For a simplicial complex $K$ it is useful to define the deleted join $K_{\Delta}^{* 2}$ in the following way. Let the vertex set of $K$ be $V$. Then the vertex set of $K_{\Delta}^{* 2}$ is $V_{1} \sqcup V_{2}$, the disjoint union of two copies of $V$. The simplices of $K_{\Delta}^{* 2}$ are pairs of simplices of $K, \sigma_{1} \subseteq V_{1}$, $\sigma_{2} \subseteq V_{2}$, such that, considered as subsets of $V$, they have $\sigma_{1} \cap \sigma_{2}=\emptyset$.

Deleted squares and joins have a natural $Z_{2}$ action by permutation. As is discussed in [6], the simplest way to prove that some topological space $X$ cannot be embedded into $\mathbb{R}^{n}$ is to show that hind $X_{\Delta}^{2} \geq n$. Similarly, for a simplicial complex $K$ it is sufficient to show that hind $K_{\Delta}^{* 2} \geq n+1$.

Now we can formulate the transversal analogues of nonembeddability theorems. 
Theorem 2. Let $X_{0}, X_{1}, \ldots, X_{m}$ be topological spaces such that for any $i=0, \ldots, m$, $\operatorname{hind}\left(X_{i}\right)_{\Delta}^{2} \geq d-m$. Suppose for each $i=0, \ldots, m$ we have a continuous map $f_{i}: X_{i} \rightarrow$ $\mathbb{R}^{d}$. Then there exist $m+1$ pairs of distinct points $x_{i}, y_{i} \in X_{i}$ such that their images $\left\{f_{i}\left(x_{i}\right), f_{i}\left(y_{i}\right)\right\}_{i=0}^{m}$ are met by some $m$-flat in $\mathbb{R}^{d}$.

Theorem 3. Let $K_{0}, K_{1}, \ldots, K_{m}$ be simplicial complexes such that, for any $i=$ $0, \ldots, m, \operatorname{hind}\left(K_{i}\right)_{\Delta}^{* 2} \geq d-m+1$. Suppose for each $i=0, \ldots, m$ we have a continuous map $f_{i}: K_{i} \rightarrow \mathbb{R}^{d}$. Then there exist $m+1$ pairs of points $x_{i}, y_{i} \in K_{i}$ with disjoint support simplices such that their images $\left\{f_{i}\left(x_{i}\right), f_{i}\left(y_{i}\right)\right\}_{i=0}^{m}$ are met by some m-flat in $\mathbb{R}^{d}$.

For example, if we consider the $d$-skeleton of a $(2 d+2)$-dimensional simplex $K=$ $\Delta_{2 d+2}^{d}$, then hind $\left(K_{\Delta}^{* 2}\right)=2 d+1$. This is shown in [6] for another definition of the index, but in fact the reasoning is the same as for the homological index.

Using this fact we can formulate the following corollary that generalizes the Van Kampen-Flores theorem from [9] and [3], which is the case $m=0$ of the following statement.

Corollary 4. If we have $m+1$ continuous maps $f_{i}: \Delta_{2 d+2}^{d} \rightarrow \mathbb{R}^{2 d+m}$, then there exist $m+1$ pairs of points $x_{i}, y_{i} \in \Delta_{2 d+2}^{d}$ with disjoint supports in each pair such that their images $\left\{f_{i}\left(x_{i}\right), f_{i}\left(y_{i}\right)\right\}_{i=0}^{m}$ are met by some $m$-flat in $\mathbb{R}^{2 d+m}$.

\section{Colored Version of Tverberg's Transversal Conjecture}

Denote $[n]=\{1,2, \ldots, n\}$.

Definition. Let set $S$ be colored into $n$ colors (i.e. mapped to $[n]$ ). Call a nonempty subset $\sigma \subseteq S$ multicolored if each color occurs in $\sigma$ no more than once.

Theorem 5. Let $0 \leq m \leq d-1$ and let $S_{0}, S_{1}, \ldots, S_{m}$ be $m+1$ finite sets in $\mathbb{R}^{d}$. Let $r_{i}$ $(i=0, \ldots, m)$ be powers of the same prime $p$. Let $k$ be the number of colors and either $k=d-m+1$ or $k<d-m+1$ and, for each $i=0, \ldots, m, r_{i} \leq(d-m) /(d-m+1-k)$. If $p \neq 2$ we require that $d-m$ is even. Let $t_{i}=2 r_{i}-1$. Let $\left|S_{i}\right|=t_{i} k$ and let each $S_{i}$ be colored in $k$ colors so that each color is used in $S_{i} t_{i}$ times. Then we can find $r_{i}$ disjoint multicolored subsets for each $i$,

$$
P_{i 1}, P_{i 2}, \ldots, P_{i r_{i}} \subseteq S_{i},
$$

so that all the sets conv $P_{i j}\left(i=0, \ldots, m, j=1, \ldots, r_{i}\right)$ can be met by a single $m$-flat.

We also formulate another version of this theorem

Theorem 6. Theorem 5 is also true when $k=d+1-m$ and for those of $r_{i}$ that equal 2 we take $t_{i}=2$ instead of 3 .

Theorems 5 and 6 are straightforward generalizations of the colored Tverberg's theorem from [12]-[14]. They generalize the colored Tverberg's theorem in the same way 
as the results from [15] and [11] and Theorem 1 generalize the noncolored Tverberg's theorem to $m$-transversals.

It will be seen from the proof that the sets $S_{i}$ can be replaced by continuous maps of simplicial complexes $K_{i}$ (defined below) to $\mathbb{R}^{d}$ and conv $P_{i j}$ can be replaced by the images of pairwise disjoint simplices of $K_{i}$. That means that in fact we prove some stronger topological versions of Theorems 5 and 6.

Similar to the colored Tverberg's conjecture, it is natural to ask whether the number $t=2 r-1$ in Theorem 5 can be made less, or even whether it can be $t=r$ when $r \neq 2$. The colored Tverberg's conjecture for $t=r \neq 2$ is only proved in [1] for $d=2$ by a nontopological method, so making $t$ less in Theorem 5 seems to be quite a hard problem.

\section{Constructions with Vector Spaces}

We use constructions from the topological proof of Tverberg's theorem [7] as they are used in [6]. We use them for the case of prime powers, following the ideas from [10].

Consider a vector space $V$ and an integer $n>0$. Let us make a definition.

Definition. Take $\mathbb{R}^{n}$ with coordinates $\left(t_{1}, \ldots, t_{n}\right)$ and consider the hyperplane $A_{n}$ given by the equation

$$
t_{1}+t_{2}+\cdots+t_{n}=1
$$

If we want to treat $A_{n}$ as a vector space, we put its origin to $(1 / n, 1 / n, \ldots, 1 / n)$.

Definition. For a vector space $V$ and an integer $n>0$ put

$$
J_{A}^{n}(V)=n V \oplus A_{n},
$$

here $n V$ is the direct sum of $n$ copies of $V$. The space $V$ can be embedded into $J_{A}^{n}(V)$ with the map

$$
v \mapsto v \oplus v \oplus \cdots \oplus v \oplus(1 / n, \ldots, 1 / n),
$$

and this gives the orthogonal decomposition

$$
J_{A}^{n}(V)=V \oplus D_{A}^{n}(V) .
$$

In fact it can be easily seen that $J_{A}^{n}(V)$ is the affine hull of the $n$-fold join $V * V * \cdots * V$.

Action of any group $G$ on the index set $[n]$ gives an action on $n V$ by permutations of the summands, it also gives an action on $A_{n}$. The summand $V$ in the decomposition $J_{A}^{n}(V)=V \oplus D_{A}^{n}(V)$ is stable under this action. If $G$ acts transitively on [n], then its representation on $D_{A}^{n}(V)$ has no trivial summands.

In what follows we consider $G=\left(Z_{p}\right)^{k}$ ( $p$ is a prime) and choose some bijection betweeN $[n]\left(n=p^{k}\right)$ and $G$. Thus $G$ acts on $[n]$ by shifts.

In this case the representation $D_{A}^{n}(V)$ has no trivial summands and (see Section 1 in Chapter IV of [5] for description of $\left.H_{G}^{*}\left(\mathrm{pt}, Z_{p}\right)\right)$ its Euler class $e\left(D_{A}^{n}(V)\right) \neq 0 \in$ $H_{G}^{*}\left(\mathrm{pt}, Z_{p}\right)$. 


\section{Topological Constructions}

Let $r=p^{k}$, let $p$ be a prime, and let $G=\left(Z_{p}\right)^{k}$. Consider the $N$-fold join $E G_{N}=$ $[r] *[r] * \cdots *[r]$. It is known that it is $(N-1)$-dimensional and $(N-2)$-connected, see [6] for a very clear explanation of these matters.

The action of $G$ on $[r]$ induces the free action of $G$ on $E G_{N}$ and the canonical map

$$
H_{G}^{*}\left(\mathrm{pt}, Z_{p}\right) \rightarrow H_{G}^{*}\left(E G_{N}, Z_{p}\right)
$$

is injective in dimesions $\leq N-1$, similar to what is done in [10].

In the proof of Theorem 1 it is crucial that the simplicial complex $E G_{N}$ is $G$ equivariantly isomorphic to the $r$-fold deleted join of the $(N-1)$-dimensional simplex, i.e.

$$
E G_{N}=\left(\Delta^{N-1}\right)_{\Delta}^{* r} .
$$

The space of the deleted join is the subset of the ordinary join $\left(\Delta^{N-1}\right)^{* r}$ consisting of affine combinations of $r$ points of $\Delta^{N-1}$,

$$
c_{1} x_{1} \oplus c_{2} x_{2} \oplus \cdots \oplus c_{r} x_{r}
$$

with pairwise disjoint supports.

In the proof of Theorem 5 we have another configuration space. Consider the following simplicial complex $K$. Let its vertex set be $S=[k] \times[t]$ and let the first factor $[k]$ denote the color of the vertex. Let the simplices of $K$ be all multicolored subsets $\sigma \subseteq S$.

Let us describe the $r$-fold deleted join $L=K_{\Delta}^{* r}$. Its vertex set is $[r] \times S$, every simplex $\sigma \subseteq L$ can considered as a union

$$
\sigma=1 \times \sigma_{1} \cup 2 \times \sigma_{2} \cup \cdots \cup r \times \sigma_{r},
$$

where $\left\{\sigma_{1}, \ldots, \sigma_{r}\right\}$ are the disjoint simplices of $K$. In [12] and [13] it is shown that the complex $L$ is $(r k-2)$-connected.

The action of $G$ on $[r]$ induces the free action of $G$ on $L$ and the canonical map

$$
H_{G}^{*}\left(\mathrm{pt}, Z_{p}\right) \rightarrow H_{G}^{*}\left(L, Z_{p}\right)
$$

is injective in dimesions $\leq r k-1$, this fact can be deduced from the Serre spectral sequence of the fibration $(E G \times L) / G \rightarrow B G$, see Section 1 in Chapter III of [5].

Next we recall Künneth's formula (see [4]) for cohomology with coefficients in some field $F$ :

$$
H^{n}(X \times Y, F)=\bigoplus_{k=0}^{n} H^{k}(X, F) \otimes H^{n-k}(Y, F),
$$

where the map from $H^{k}(X, F) \times H^{n-k}(Y, F)$ to $H^{n}(X \times Y, F)$ is given by the $\times$-product.

We formulate the following simple statement as a separate lemma, which will be required in the proofs below. 
Lemma 7. If the space $Y$ is connected then some inclusion $i: X=X \times\left\{y_{0}\right\} \rightarrow X \times Y$ gives the map

$$
i^{*}: \bigoplus_{k=0}^{n} H^{k}(X, F) \otimes H^{n-k}(Y, F) \rightarrow H^{n}(X, F),
$$

which is the projection onto the $n$th summand of $\bigoplus_{k=0}^{n} H^{k}(X, F) \otimes H^{n-k}(Y, F)$, having in mind that $H^{0}(Y, F)=F$.

The definition of equivariant cohomology immediately gives for $G_{1}$-space $X$ and $G_{2}$-space $Y$ the equivariant Künneth's formula:

$$
H_{G_{1} \times G_{2}}^{n}(X \times Y, F)=\bigoplus_{k=0}^{n} H_{G_{1}}^{k}(X, F) \otimes H_{G_{2}}^{n-k}(Y, F) .
$$

If we consider two (equivariant) oriented vector bundles $\xi \rightarrow X$ and $\eta \rightarrow Y$, then the space $\xi \times \eta$ is a vector bundle over $X \times Y$ and we have the multiplicative rule for the Euler class

$$
e(\xi \times \eta)=e(\xi) \times e(\eta) .
$$

When the bundles are over the same space and we take their fiberwise direct sum we have (we use simple multiplicative notation for the cup product)

$$
e(\xi \oplus \eta)=e(\xi) e(\eta) .
$$

\section{Lemma about the Grassmann Variety}

Consider the canonical bundle over the Grassmann variety $\gamma \rightarrow G_{d}^{d-m}$. In the case $p=2$ we consider the variety of nonoriented $(d-m)$-subspaces, and for odd $p$ we consider the variety of oriented subspaces. If we have to distinguish between these cases we denote the oriented Grassmann variety by $G_{d}^{+d-m}$.

Lemma 8. For the Euler class $e(\gamma)$ modulo $p$ the following holds:

$$
e(\gamma)^{m} \neq 0 \in H^{m(d-m)}\left(G_{d}^{d-m}, Z_{p}\right),
$$

if $p=2$ or $d-m$ is even.

The case of this lemma for $p=2$ is proved in [2]. The case for $p$ odd and $d$ odd is proved in [15].

Proof. Here we give the proof for the case of odd $p$ and the oriented Grassmann variety $G_{d}^{+d-m}$. Denote $l=d-m$.

We use the method of test sections. That means that to prove that an $N$-dimensional oriented vector bundle $\eta$ over an $N$-dimensional oriented manifold $M$ has nonzero Euler class we should do the following. Take some section of this bundle and consider its zero 
points. At each zero point consider some of its vicinity with properly oriented coordinates $x_{1}, \ldots, x_{N}$, the bundle here can be considered trivial with properly oriented coordinates $u_{1}, \ldots, u_{N}$. The section is locally given by

$$
\begin{aligned}
u_{1} & =h_{1}\left(x_{1}, \ldots, x_{N}\right) \\
& \vdots \\
u_{N} & =h_{N}\left(x_{1}, \ldots, x_{N}\right),
\end{aligned}
$$

and the index of this zero point is $\operatorname{sgn} \operatorname{det}\left(\partial h_{i} / \partial x_{j}\right)$. If all zero points of the section are transversal (i.e. their indices are nonzero) the Poincaré duality (see [4]) shows that $e(\eta)=s w(M)$, where $s$ is the sum of indices of all zero points and $w(M)$ is the fundamental $N$-dimensional class of $M$.

Now we have an $m l$-dimensional manifold $G_{d}^{+l}$ and an $m l$-dimensional bundle $\gamma \oplus$ $\cdots \oplus \gamma=m \gamma$ ( $m$-fold direct sum of $\gamma)$.

Take $m$ orthonormal vectors $f_{1}, f_{2}, \ldots, f_{m} \in \mathbb{R}^{d}$ and consider the sections of $\gamma$ that are formed by orthogonal projections of $f_{i}$ to the corresponding $l$-subspace $L \in$ $G_{d}^{+l}$. We denote these sections by $f_{1}, \ldots, f_{m}$ too. Together they form a section $f=$ $\left(f_{1}, f_{2}, \ldots, f_{m}\right)$ of $m \gamma$. As is easily seen, $f$ has two zero points in $G_{d}^{+l}$. They correspond to the $l$-subspace $L$ that is orthogonal to vectors $f_{i}$ with two possible orientations.

Choose the orthonormal base $e_{1}, \ldots, e_{l} \in L$. Consider the case when $e_{1} \wedge \cdots \wedge e_{l} \wedge$ $f_{1} \wedge \cdots \wedge f_{m}$ is positive. The elements of $G_{d}^{+l}$ close to $L$ can be uniquely described by the base of form

$$
\begin{aligned}
e_{1}^{\prime} & =e_{1}+x_{11} f_{1}+\cdots+x_{1 m} f_{m} \\
& \vdots \\
e_{l}^{\prime} & =e_{l}+x_{l 1} f_{1}+\cdots+x_{l m} f_{m},
\end{aligned}
$$

and the orientation of $G_{d}^{+l}$ is given by (row-wise product)

$$
\omega=d x_{11} \wedge d x_{12} \wedge \cdots \wedge d x_{1 m} \wedge \cdots \wedge d x_{l 1} \wedge \cdots \wedge d x_{l m} .
$$

The section $f$ of $\gamma$ locally has the form as a map $G_{d}^{+l} \rightarrow \mathbb{R}^{l m}$ (columnwise order),

$$
\left(x_{11}, x_{21}, \ldots, x_{l 1}\right) \oplus \cdots \oplus\left(x_{1 m}, x_{2 m}, \ldots, x_{l m}\right) .
$$

We see that this is a transversal zero point and its index equals the sign of permutation that is induced by the change of row-wise to columnwise order, denote it by $I= \pm 1$.

Now if we consider the base of $L$ with a different orientation, we have the same index for section $f^{\prime}=\left(f_{2}, f_{1}, \ldots, f_{m}\right)$. Then transposing $f_{1}$ and $f_{2}$ does not change the index, since $l$ is even. Thus the second point has the same index $I$.

Finally we have that $e(m \gamma)=e(\gamma)^{m}=I\left[G_{d}^{+l}\right]= \pm 2\left[G_{d}^{+l}\right]$, which is not zero modulo odd primes. 


\section{Proof of Theorem 1}

Put, for all $i=0, \ldots, m G_{i}=\left(Z_{p}\right)^{k_{i}}, N_{i}=\left(r_{i}-1\right)(d-m+1)+1$. Denote $l=d-m$. The Grassmann variety will be oriented for odd $p$.

In this theorem we need $r_{i}$-tuples of points in each $\Delta_{i}$ with pairwise disjoint supports, so it is natural to consider the $r_{i}$-fold deleted joins $E G_{N_{i}}=\left(\Delta^{N_{i}-1}\right)_{\Delta}^{* r_{i}}$ as proper configuration spaces, as it is frequently done in [6].

For any $l$-dimensional linear subspace $V \subseteq \mathbb{R}^{d}$ consider the deleted join map

$$
h_{i, V}: E G_{N_{i}} \rightarrow J_{A}^{r_{i}}(V),
$$

note that the space to the right contains the $r_{i}$-fold join of $V$.

The maps $h_{i, V}$ are equivariant under the actions of $G_{i}$. Take the cartesian product of these maps to get the $\left(G=G_{0} \times \cdots \times G_{m}\right)$-equivariant map

$$
h_{0, V} \times \cdots \times h_{m, V}: E G_{N_{0}} \times \cdots \times E G_{N_{m}} \rightarrow J_{A}^{r_{0}}(V) \oplus \cdots \oplus J_{A}^{r_{m}}(V) .
$$

Considering the dependence of this map on $V \in G_{d}^{l}$ we get an equivariant section of the bundle

$$
\xi=J_{A}^{r_{0}}(\gamma) \oplus \cdots \oplus J_{A}^{r_{m}}(\gamma) \rightarrow E G_{N_{0}} \times \cdots \times E G_{N_{m}} \times G_{d}^{l} .
$$

Now consider the decomposition

$$
\xi=D_{A}^{r_{0}}(\gamma) \oplus \cdots \oplus D_{A}^{r_{m}}(\gamma) \oplus(m+1) \gamma
$$

and with the diagonal embedding $\gamma \rightarrow(m+1) \gamma$ we also have the decomposition $(m+1) \gamma=\gamma_{\Delta} \oplus \eta$. From here on we choose some isomorphism between $\eta$ and $m \gamma$, in matrix form it may be given by some orthogonal basis in the orthogonal complement of the vector $(1, \ldots, 1)$ in $\mathbb{R}^{m+1}$. Thus denote

$$
\zeta=D_{A}^{r_{0}}(\gamma) \oplus \cdots \oplus D_{A}^{r_{m}}(\gamma) \oplus \eta \rightarrow E G_{N_{0}} \times \cdots \times E G_{N_{m}} \times G_{d}^{l} .
$$

Note that if the section constructed above in projection to $\zeta$ has a zero, then we are done. Indeed, in this case we have some $V$ and $m+1$ points $y_{i} \in E G_{N_{i}}$. Now remember that every $E G_{N_{i}}$ is a deleted join, that means that, for all $i=0, \ldots, m, y_{i}$ is an affine combination

$$
y_{i}=c_{i 1} x_{i 1} \oplus \cdots \oplus c_{i r_{i}} x_{i r_{i}}
$$

of points in $\Delta_{i}$ with disjoint supports. The condition that the section has a zero means that, for all $i$ and $j, c_{i j}=1 / r_{i}$ and the projections of $f_{i}\left(x_{i j}\right)$ to $V$ coincide and give a point $v$. Taking the affine $m$-subspace of $\mathbb{R}^{d}$ orthogonal to $V$ and passing through $v$ we get what we need.

Finally, we have to show that the equivariant Euler class

$$
e(\zeta) \in H_{G}^{*}\left(E G_{N_{0}} \times \cdots \times E G_{N_{m}} \times G_{d}^{l}, Z_{p}\right)
$$

is nonzero. Note that by Lemma 8 we have $e(\eta) \neq 0 \in H^{*}\left(G_{d}^{l}, Z_{p}\right)$. Also note that any embedding with fixed $V \in G_{d}^{l}$,

$$
g: E G_{N_{0}} \times \cdots \times E G_{N_{m}} \rightarrow E G_{N_{0}} \times \cdots \times E G_{N_{m}} \times G_{d}^{l},
$$


induces the bundle

$$
\beta=g^{*}\left(D_{A}^{r_{0}}(\gamma) \oplus \cdots \oplus D_{A}^{r_{m}}(\gamma)\right) \rightarrow E G_{N_{0}} \times \cdots \times E G_{N_{m}},
$$

which is the cartesian product of $G_{i}$-bundles over $E G_{N_{i}}$, arising from the representations of $G_{i}$ in $D_{A}^{r_{i}}(V)$. Since $\operatorname{dim} D_{A}^{r_{i}}(V)=N_{i}-1$, the construction of $E G_{N_{i}}$ implies that each of these bundles has a nonzero Euler class in $H^{*}\left(E G_{N_{i}}, Z_{p}\right)$ and, by the multiplicative rule and Künneth's formula,

$$
e(\beta) \neq 0 \in H_{G}^{*}\left(E G_{N_{0}} \times \cdots \times E G_{N_{m}}, Z_{p}\right) .
$$

Using Künneth's formula for $\left(E G_{N_{0}} \times \cdots \times E G_{N_{m}}\right) / G \times G_{d}^{l}$ and Lemma 7 we see that

$$
e\left(D_{A}^{r_{0}}(\gamma) \oplus \cdots \oplus D_{A}^{r_{m}}(\gamma)\right)=e(\beta) \times 1+\sum u \times v,
$$

where $u \in H_{G}^{*}\left(E G_{N_{0}} \times \cdots \times E G_{N_{m}}, Z_{p}\right), v \in H^{*}\left(G_{d}^{l}, Z_{p}\right)$ and all $v$ have $\operatorname{dim} v>0$.

Now by the multiplicative rule

$$
e(\zeta)=e\left(D_{A}^{r_{0}}(\gamma) \oplus \cdots \oplus D_{A}^{r_{m}}(\gamma)\right) e(\eta)=e(\beta) \times e(\eta)+\sum u \times v e(\eta) \neq 0
$$

in the cohomology $H_{G}^{*}\left(E G_{N_{0}} \times \cdots \times E G_{N_{m}} \times G_{d}^{l}, Z_{p}\right)$.

\section{Proof of Theorems 2 and 3}

In the following proofs we also denote $l=d-m$.

Proof of Theorem 2. Consider some $l$-subspace $V$ of $\mathbb{R}^{d}$ and denote the orthogonal projection to $V$ by $\pi_{V}$. For any $i=0, \ldots, m$ consider the map $s_{i, V}:\left(X_{i}\right)_{\Delta}^{2} \rightarrow V$ given by

$$
s_{i, V}(x, y)=\pi_{V}\left(f_{i}(x)\right)-\pi_{V}\left(f_{i}(y)\right) .
$$

This is a $Z_{2}$-equivariant map if $Z_{2}$ acts on $V$ antipodally. These maps for $i=0, \ldots, m$ form a map

$$
s_{V}:\left(X_{0}\right)_{\Delta}^{2} \times \cdots \times\left(X_{m}\right)_{\Delta}^{2} \rightarrow V \oplus \cdots \oplus V=(m+1) V .
$$

Also consider the maps $t_{i, V}:\left(X_{i}\right)_{\Delta}^{2} \rightarrow V$ given by

$$
t_{i, V}(x, y)=\pi_{V}\left(f_{i}(x)\right)+\pi_{V}\left(f_{i}(y)\right),
$$

the product of these maps gives a map $t_{V}:\left(X_{0}\right)_{\Delta}^{2} \times \cdots \times\left(X_{m}\right)_{\Delta}^{2} \rightarrow(m+1) V$. The space $(m+1) V$ can be decomposed into its diagonal and its orthogonal complement $(m+1) V=V_{\Delta} \oplus W$. So we consider the composition of $t_{V}$ with the projection to $W$ and get the map $u_{V}:\left(X_{0}\right)_{\Delta}^{2} \times \cdots \times\left(X_{m}\right)_{\Delta}^{2} \rightarrow W$.

Then we take the map

$$
s_{V} \oplus u_{V}:\left(X_{0}\right)_{\Delta}^{2} \times \cdots \times\left(X_{m}\right)_{\Delta}^{2} \rightarrow(m+1) V \oplus W,
$$


this map can be considered as a section of the vector bundle $\xi \oplus \eta=(m+1) \gamma \oplus m \gamma$ over $\left(X_{0}\right)_{\Delta}^{2} \times \cdots \times\left(X_{m}\right)_{\Delta}^{2} \times G_{d}^{l}$. Here we again choose some isomorphism between the bundle $\eta$ arising from spaces $W$ and $m \gamma$.

The constructed section is equivariant with respect to the action of $G=\left(Z_{2}\right)^{m+1}$ on $\xi \oplus \eta=(m+1) \gamma \oplus m \gamma$ by antipodal maps on the former $m+1$ summands.

It is easy to see that if this section has a zero, then the projections of the corresponding points $\left\{f_{i}\left(x_{i}\right), f_{i}\left(y_{i}\right)\right\}_{i=0}^{m}$ to $V$ coincide and the theorem is proved.

All we have to do is to prove that the Euler class of this bundle is nonzero modulo 2. By Lemma 8,

$$
e(\eta)=e(\gamma)^{m} \neq 0 \in H^{m(d-m)}\left(G_{d}^{l}, Z_{2}\right) .
$$

Taking some fixed $V \in G_{d}^{l}$ we have a map

$$
\left(X_{0}\right)_{\Delta}^{2} \times \cdots \times\left(X_{m}\right)_{\Delta}^{2} \rightarrow\left(X_{0}\right)_{\Delta}^{2} \times \cdots \times\left(X_{m}\right)_{\Delta}^{2} \times G_{d}^{l}
$$

that induces a bundle $\xi^{\prime}$ over $\left(X_{0}\right)_{\Delta}^{2} \times \cdots \times\left(X_{m}\right)_{\Delta}^{2}$. By Künneth's formula, the multiplicative rule for the Euler class, and the index property of $\left(X_{i}\right)_{\Delta}^{2}$ we have

$$
e\left(\xi^{\prime}\right)=u^{l} \times \cdots \times u^{l} \neq 0 \in H_{G}^{l(m+1)}\left(\left(X_{0}\right)_{\Delta}^{2} \times \cdots \times\left(X_{m}\right)_{\Delta}^{2}, Z_{2}\right) .
$$

This means that by Künneth's formula the Euler class $e(\xi)$ in $H_{G}^{*}\left(\left(X_{0}\right)_{\Delta}^{2} \times \cdots \times\left(X_{m}\right)_{\Delta}^{2} \times\right.$ $G_{d}^{l}, Z_{2}$ ) has the form $e(\xi)=e\left(\xi^{\prime}\right) \times 1+\sum a \times b$ (by Lemma 7), where $\operatorname{dim} b>1$ always. Then by the multiplicative rule we see that $e(\xi \oplus \eta)=e\left(\xi^{\prime}\right) \times e(\eta)+\sum a \times b e(\eta)$ $\neq 0$.

Proof of Theorem 3. The proof is similar to the previous one, so we only show the differences.

Instead of maps $s_{i, V}$ and $t_{i, V}$ we consider the map $r_{i, V}:\left(K_{i}\right)_{\Delta}^{* 2} \rightarrow V \oplus V \oplus L$, where $L$ is a one-dimensional linear space. This map is given by

$$
r_{i, V}(t x \oplus(1-t) y)=t \pi_{V}\left(f_{i}(x)\right) \oplus(1-t) \pi_{V}\left(f_{i}(y)\right) \oplus\left(t-\frac{1}{2}\right) .
$$

This map is obviously equivariant with respect to the $Z_{2}$-action on $\left(K_{i}\right)_{\Delta}^{* 2}$ and its action on $V \oplus V \oplus L$ that permutes two summands $V$ and acts antipodally on $L$.

The considered space $V \oplus V \oplus L$ may be decomposed into $U_{i} \oplus W_{i}$, where $Z_{2}$ acts antipodally on the $(l+1)$-dimensional space $U_{i}$ and trivially on the $l$-dimensional space $W_{i}$.

We sum up the maps $r_{i}$ to have the map

$$
r_{V}:\left(K_{0}\right)_{\Delta}^{* 2} \times \cdots \times\left(K_{m}\right)_{\Delta}^{* 2} \rightarrow\left(U_{0} \oplus \cdots \oplus U_{m}\right) \oplus\left(W_{0} \oplus \cdots \oplus W_{m}\right)=U \oplus W .
$$

Then we decompose $W=W^{\prime} \oplus V_{\Delta}$ by the diagonal map $V \rightarrow W$ and project $r_{V}$ to $U \oplus W^{\prime}$. This map can be regarded as the $G=\left(Z_{2}\right)^{m+1}$ equivariant section of the bundle $\xi \oplus \eta$, where $\xi$ arises from $U$ and $\eta$ arises from $W^{\prime}$.

By Lemma $8, e(\eta) \neq 0$ and the rest of the proof is similar to the proof of Theorem 2. 


\section{Proof of Theorems 5 and 6}

Proof of Theorem 5. We prove this theorem in the same way as we prove Theorem 1.

Let $r_{i}=p^{k_{i}}$ and put, for all $i=0, \ldots, m, G_{i}=\left(Z_{p}\right)^{k_{i}}$. For each of the set $S_{i}$ we take the simplicial complex of multicolored subsets $K_{i}$ (as described above) and its $r_{i}$-fold deleted join $L_{i}=K_{i \Delta}^{* r_{i}}$ with appropriate $G_{i}$-action.

Denote $l=d-m$. The Grassmann variety will be oriented for odd $p$.

For any $l$-dimensional linear subspace $V \subseteq \mathbb{R}^{d}$ consider the natural piecewise linear (or simply continuous for the topological version) projection $K_{i} \rightarrow V$ and its $r_{i}$-fold join

$$
h_{i, V}: L_{i} \rightarrow J_{A}^{r_{i}}(V)
$$

The maps $h_{i, V}$ are equivariant under the actions of $G_{i}$. Take the cartesian product of these maps to get the $\left(G=G_{0} \times \cdots \times G_{m}\right)$-equivariant map

$$
h_{0, V} \times \cdots \times h_{m, V}: L_{0} \times \cdots \times L_{m} \rightarrow J_{A}^{r_{0}}(V) \oplus \cdots \oplus J_{A}^{r_{m}}(V) .
$$

Now considering the dependence of this map on $V \in G_{d}^{l}$ we get an equivariant section of the bundle

$$
\xi=J_{A}^{r_{0}}(\gamma) \oplus \cdots \oplus J_{A}^{r_{m}}(\gamma) \rightarrow L_{0} \times \cdots \times L_{m} \times G_{d}^{l}
$$

Consider the decomposition

$$
\xi=D_{A}^{r_{0}}(\gamma) \oplus \cdots \oplus D_{A}^{r_{m}}(\gamma) \oplus(m+1) \gamma
$$

and with the diagonal embedding $\gamma \rightarrow(m+1) \gamma$ we also have the decomposition $(m+1) \gamma=\gamma_{\Delta} \oplus \eta$. As in the previous proofs we identify $\eta=m \gamma$.

Thus we denote

$$
\zeta=D_{A}^{r_{0}}(\gamma) \oplus \cdots \oplus D_{A}^{r_{m}}(\gamma) \oplus \eta \rightarrow L_{0} \times \cdots \times L_{m} \times G_{d}^{l} .
$$

If the section constructed above in projection to $\zeta$ has a zero, we are done as in the proof of Theorem 1.

Finally we have to show that the equivariant Euler class

$$
e(\zeta) \in H_{G}^{*}\left(L_{0} \times \cdots \times L_{m} \times G_{d}^{l}, Z_{p}\right)
$$

is nonzero. Note that by Lemma 8 we have $e(\eta) \neq 0 \in H^{*}\left(G_{d}^{l}, Z_{p}\right)$, and any embedding with fixed $V \in G_{d}^{l}$

$$
g: L_{0} \times \cdots \times L_{m} \rightarrow L_{0} \times \cdots \times L_{m} \times G_{d}^{l},
$$

induces the bundle

$$
\beta=g^{*}\left(D_{A}^{r_{0}}(\gamma) \oplus \cdots \oplus D_{A}^{r_{m}}(\gamma)\right) \rightarrow L_{0} \times \cdots \times L_{m},
$$

which is the cartesian product of $G_{i}$-bundles over $L_{i}$, arising from the representation of $G_{i}$ in $D_{A}^{r_{i}}(V)$. Since $\operatorname{dim} D_{A}^{r_{i}}(V)=\left(r_{i}-1\right)(d-m+1)$, the construction of $L_{i}$ and the conditions of the theorem imply that

$$
r_{i} k-1 \geq\left(r_{i}-1\right)(d-m+1)
$$


and each of summand bundles has a nonzero Euler class in $H^{*}\left(L_{i}, Z_{p}\right)$. Thus by Künneth's formula and the multiplicative rule for the Euler class,

$$
e(\beta) \neq 0 \in H_{G}^{*}\left(L_{0} \times \cdots \times L_{m}, Z_{p}\right) .
$$

Using Künneth's formula for $\left(L_{0} \times \cdots \times L_{m}\right) / G \times G_{d}^{l}$ and Lemma 7 we see that

$$
e\left(D_{A}^{r_{0}}(\gamma) \oplus \cdots \oplus D_{A}^{r_{m}}(\gamma)\right)=e(\beta) \times 1+\sum u \times v,
$$

where $u \in H_{G}^{*}\left(L_{0} \times \cdots \times L_{m}, Z_{p}\right), v \in H^{*}\left(G_{d}^{l}, Z_{p}\right)$ and all $v$ have $\operatorname{dim} v>0$.

Now by the multiplicative rule for the Euler class,

$$
e(\zeta)=e\left(D_{A}^{r_{0}}(\gamma) \oplus \cdots \oplus D_{A}^{r_{m}}(\gamma)\right) e(\eta)=e(\beta) \times e(\eta)+\sum u \times v e(\eta) \neq 0
$$

in the cohomology $H_{G}^{*}\left(L_{0} \times \cdots \times L_{m} \times G_{d}^{l}, Z_{p}\right)$.

Proof of Theorem 6. Here we give only the differences from the previous proof.

Consider $r_{i}=t_{i}=2$. In this case $S_{i}$ has a natural $G_{i}$-action $\left(G_{i}=Z_{2}\right)$ that exchanges every two vertices with the same color. This gives free $G_{i}$-action on $K_{i}$ itself, so we can consider $L_{i}=K_{i}$. Also note that $K_{i}$ is the boundary of the $k$-dimensional cross-polytope, so $K_{i}$ is a $(k-1)$-dimensional $((d-m)$-dimensional) sphere.

In the case $r_{i}=2$ instead of the map

$$
h_{i, V}: L_{i} \rightarrow J_{A}^{2}(V)
$$

we consider the map

$$
h_{i, V}: K_{i} \rightarrow V \oplus V
$$

given by $x \in K_{i} \mapsto \pi(x) \oplus \pi(-x)$, where by the minus sign we denote the action of $Z_{2}$. This is a $Z_{2}$-equivariant map when $Z_{2}$ permutes the summands of $V \oplus V$.

Instead of the projection $J_{A}^{2}(V) \rightarrow D_{A}^{2}(V)$ we consider the projection $V \oplus V \rightarrow V$ given by $v_{1} \oplus v_{2} \mapsto v_{1}-v_{2}$. The codomain space $V$ here has an antipodal $Z_{2}$-action and a point $x \in K_{i}$ maps to zero in $V$ iff the projections of $x$ and $-x$ to $V$ coincide.

The space $V$ has a nonzero equivariant Euler class in $H_{Z_{2}}^{d-m}\left(\mathrm{pt}, Z_{2}\right)$ and in $H_{Z_{2}}^{d-m}\left(K_{i}, Z_{2}\right)$. The rest of the above proof works without change.

\section{Acknowledgements}

I thank R.T. Živaljević and S.T. Vrećica for useful discussions of these results. I also thank V.L. Dol'nikov for constant attention to my work and helpful advice.

\section{References}

1. Barany, I., and Larman, D.G. A colored version of Tverberg's theorem. J. London Math. Soc., 45(2) (1992), $314-320$ 
2. Dol'nikov, V.L. Common transversals for families of sets in $\mathbb{R}^{n}$ and connections between theorems of Helly and Borsuk. (In Russian.) Dokl. Akad. Nauk USSR, 297(4) (1987), 777-780.

3. Flores, A. Über $n$-dimensionale Komplexe, die im $R_{2 n+1}$ absolut selbstverschlungen sind. Ergeb. Math. Kolloq., 6 (1932/1934), 4-7.

4. Hatcher, A. Algebraic Topology. Cambridge University Press, Cambridge, 2002

5. Hsiang Wu Yi. Cohomology Theory of Topological Transformation Groups. Springer-Verlag, Berlin, 1975.

6. Matoušek, J. Using the Borsuk-Ulam Theorem. Springer-Verlag, Berlin, 2003

7. Tverberg, H. A generalization of Radon's theorem. J. London Math. Soc., 41 (1966), 123-128.

8. Tverberg, H., and Vrećica, S. On generalizations of Radon's theorem and the ham sandwich theorem. European J. Combin., 14 (1993), 259-264

9. Van Kampen, R.E. Komplexe in euklidischen Räumen. Abh. Math. Sem. Univ. Hamburg, 9 (1932), 72-78.

10. Volovikov, A. Yu. On a topological generalization of the Tverberg theorem. Math. Notes, 59(3) (1996), 324-326

11. Vrećica, S.T. Tverberg's conjecture. Discrete Comput. Geom., 29 (2003), 505-510

12. Vrećica, S.T., and Živaljević, R.T. The colored Tverberg's problem and complexes of injective functions. J. Combin. Theory Ser. A, 61(2) (1992), 309-318.

13. Vrećica, S.T., and Živaljević, R.T. New cases of the colored Tverberg theorem. In Jerusalem Combinatorics ' 93 (ed. by H. Barcelo and G. Kalai), pp. 325-334. Contemporary Mathematics. American Mathematical Society, Providence, RI, 1994.

14. Živaljević, R.T. User's guide to equivariant methods in combinatorics. Publ. Inst. Math., 64(78) (1998), $107-132$

15. Živaljević, R.T. The Tverberg-Vrećica problem and the combinatorial geometry on vector bundles. Israel J. Math., 111 (1999), 53-76

Received January 11, 2007, and in revised form March 13, 2007, and April 2, 2007.

Online publication August 30, 2007. 